\title{
On a Class of Dual Model with Divided Threshold
}

\author{
Yuzhen Wen \\ School of Mathematical Sciences, Qufu Normal University, Qufu, China \\ E-mail:wenyzhen@163.com \\ Received January 24, 2011; revised March 28, 2011; accepted April 1, 2011
}

\begin{abstract}
In this paper, we consider the dual of the generalized Erlang (n) risk model under a threshold dividend strategy. We derive an integro-differential equation satisfied by the expectation of the discounted dividends until ruin. The case when profits follow an exponential distribution is solved.
\end{abstract}

Keywords: Threshold Strategy, Dual Risk Model, Generalized Erlang (n) Risk Process

\section{Introduction}

In recent years, a few interesting results have been obtained on a model which is dual to the classical insurance risk model. See Avanzi et al.[1] Avanzi and Gerber [2,3] and A. C. Y. Ng [4] for example. In this model, the surplus at time $t$ is

$$
U(t)=u-c t+\sum_{k=1}^{N(t)} Z_{k}=u-c t+S(t), t \geq 0 .
$$

where $u$ and $c$ are constants, $u$ is the initial surplus, $c>0$ is the rate of expenses, $S(t)=\sum_{k=1}^{N(t)} Z_{k}$ is the aggregate positive gains process and $\left\{Z_{k}: k=1,2, \cdots\right\}$ is a sequence of independent and identically distributed claim amount nonnegative random variables with a common probability density function $p(y), y>0$. The ordinary renewal process $\{N(t), t \geq 0\}$ denotes the number of gains up to time $t$ with

$$
N(t)=\max \left\{k \geq 1: W_{1}+W_{2}+\cdots+W_{k} \leq t\right\}
$$

where the i.i.d gains waiting times $W_{i}$ have a common generalized Erlang (n) distribution, i.e. the $W_{i}^{\prime} s$ are distributed as the sum of $\mathrm{n}$ independent and exponentially distributed random variables:

$$
W_{i}=\xi_{1}+\xi_{2}+\cdots+\xi_{n}, i=1,2, \cdots, n,
$$

where $\xi_{j}(j=1,2, \cdots, n)$ may have different exponential parameters $\lambda_{j}>0$. Furthermore, we assume that $\left\{W_{i}\right\}_{i \geq 1}$ and $\left\{Z_{i}\right\}_{i \geq 1}$ are independent. In this model, the expected increase of the surplus per unit time is $E\left(X_{1}\right)\left(>c E\left(W_{1}\right)\right)$ and is assumed to be positive.

In this model, the premium rate is negative, causing the surplus to decrease. Claims, on the other hand, cause the surplus to increase. Thus the premium rate should be viewed as an expense rate and claims should be viewed as profits or gains. Though not very popular in insurance mathematics, this model has appeared in various literature (see Cramer [5], Seal [6], Takcs [7] and the references cited therein. In Avanzi et al. [1], the authors studied the expected total discounted dividends until ruin for the dual model under the barrier strategy by means of integro-differential equations. In [8] the authors consider a Sparre Andersen risk process that is perturbed by an independent diffusion process in which claim inter-arrival times have a generalized Erlang (n) distribution.

In this paper, we will study the expectation of the discounted dividends until ruin. We get integro-differential equation of the expectation of the discounted dividends until ruin. We also get the the expectation of the discounted dividends until ruin when profits follow an exponential distribution.

\section{Main Result}

We now consider a threshold dividend strategy. When $U(t)$ is below $b$, no dividends are paid and the surplus decreases at the original rate $c_{1}$. When $U(t)$ is above $b$, the surplus would decrease at a different rate $c_{2}\left(>c_{1}\right)$ and dividends are paid at rate $c_{2}-c_{1}$ Then $U(t)$ can be expressed by

$$
\mathrm{d} U(t)=\left\{\begin{array}{l}
-c_{2} \mathrm{~d} t+\mathrm{d} S(t), \quad U(t)>b ; \\
-c_{1} \mathrm{~d} t+\mathrm{d} S(t), \quad b \geq U(t) \geq 0 ;
\end{array} \quad(t \geq 0)\right.
$$

Let

$$
T=\inf \{t \geq 0: U(t) \leq 0\}
$$


( $T=\infty$ if ruin does not occur) be the time of ruin and $I(A)$ be equal to 1 if event $A$ occurs and 0 otherwise. The total discounted dividends until ruin is

$$
D(b)=\left(c_{2}-c_{1}\right) \int_{0}^{T} e^{-\delta t} I(U(t)>b) \mathrm{d} t
$$

where $\delta>0$ is the force of interest for valuation. Let

$$
V(u ; b)=E[D(b) \mid U(0)=u]
$$

denote the expectation of the discounted dividends until ruin, if the threshold dividend strategy with parameter $b$ is applied.

Since $U(t)$ have different paths for $0 \leq U(t) \leq b$ and $U(t)>b$, we define

$$
V(u ; b)=\left\{\begin{array}{lr}
V_{1}(u ; b), & b \geq u \geq 0, \\
V_{2}(u ; b), & u>b .
\end{array}\right.
$$

The following theorem provides integro-differential equations for the expectation of the discounted dividends of $V(u ; b)$.

Theorem 2.1 The expectation of the discounted dividends of $V(u ; b)$ satisfy the following integro-differential equations: when $0<u<b$ we have

$$
\begin{aligned}
& \prod_{i=1}^{n}\left[\left(1+\frac{\delta}{\lambda_{i}}\right) I+\frac{c_{1}}{\lambda_{i}} \frac{\mathrm{d}}{\mathrm{d} u}\right] V_{1}(u ; b) \\
& =\int_{0}^{b-u} V_{1}(u+y ; b) p(y) \mathrm{d} y+\int_{b-u}^{\infty} V_{2}(u+y ; b) p(y) \mathrm{d} y
\end{aligned}
$$

when $u>b$, we have

$$
\begin{aligned}
& \prod_{i=1}^{n}\left[\left(1+\frac{\delta}{\lambda_{i}}\right) I+\frac{c_{2}}{\lambda_{i}} \frac{\mathrm{d}}{\mathrm{d} u}\right] V_{2}(u ; b) \\
& =\int_{0}^{\infty} V_{2}(u+y ; b) p(y) \mathrm{d} y+\sum_{i=1}^{n} \prod_{j=i}^{n} \frac{\lambda_{j+1}+\delta}{\lambda_{j}}\left(c_{2}-c_{1}\right)
\end{aligned}
$$

where $I$ is the identity operator.

Proof. Let $S_{0}=0$ and $S_{j}=\xi_{1}+\xi_{2}+\cdots+\xi_{j}$ for $j=1,2, \cdots, n-1$ Define

$$
V_{i, j}(u ; b)=E\left[D(b) \mid S_{j}=t, U(0)=u\right]
$$

with $V_{i, 0}(u)=V_{i}(u)$ for $i=1,2$.

We first consider the case when $u>b$. We consider the infinitesimal interval from $S_{j}$ to $S_{j}+\mathrm{d} t$. For $j=0,1, \cdots, n-2$, we have

$$
\begin{aligned}
V_{2, j}(u ; b)=e^{-\delta d t}\{ & P\left(\xi_{j+1}>\mathrm{d} t\right)\left[E\left[V_{2, j}\left(u-c_{2} \mathrm{~d} t ; b\right)\right]\right. \\
& \left.\left.+P\left(\xi_{j+1} \leq \mathrm{d} t\right) E\left[V_{2, j+1}\left(u-c_{2} \mathrm{~d} t ; b\right)\right]\right]\right\} \\
& +\left(c_{2}-c_{1}\right) \frac{1-e^{-\delta d t}}{\delta} .
\end{aligned}
$$

Note that $e^{-\delta \mathrm{d} t}=1-\delta \mathrm{d} t+o(\mathrm{~d} t)$. Also we have

$$
\begin{aligned}
& P\left(\xi_{j+1}>\mathrm{d} t\right)=1-\lambda_{j+1} \mathrm{~d} t+o(\mathrm{~d} t), \\
& P\left(\xi_{j+1} \leq \mathrm{d} t\right)=\lambda_{j+1} \mathrm{~d} t+o(\mathrm{~d} t), \\
& E\left[V_{2, j}\left(u-c_{2} \mathrm{~d} t ; b\right)\right] \\
& =V_{2, j}(u ; b)-c_{2} \frac{\mathrm{d} V_{2, j}(u ; b)}{\mathrm{d} u} \mathrm{~d} t+o(\mathrm{~d} t)
\end{aligned}
$$

Substituting these formulas into (5), subtracting $V_{2, j}(u ; b)$ from both sides, interpreting $\mathrm{d} t$ and $o(\mathrm{~d} t)$ terms, canceling common factors and letting $\mathrm{d} t \rightarrow 0$, we have

$$
\begin{aligned}
& \lambda_{j+1} V_{2, j+1}(u ; b)= \\
& {\left[\left(\lambda_{j+1}+\delta\right) I+c_{2} \frac{\mathrm{d}}{\mathrm{d} u}\right] V_{2, j}(u ; b)-\left(c_{2}-c_{1}\right)}
\end{aligned}
$$

for $j=0,1, \cdots, n-2$. Similarly for $j=n-1$, we have

$$
\begin{aligned}
& {\left[\left(\lambda_{n}+\delta\right) I+c_{2} \frac{\mathrm{d}}{\mathrm{d} u}\right] V_{2, n-1}(u ; b)} \\
& =\lambda_{n} \int_{0}^{\infty} V_{2}(u+y ; b) p(y) \mathrm{d} y+\left(c_{2}-c_{1}\right) .
\end{aligned}
$$

Thus we have

$$
\begin{aligned}
& \prod_{i=1}^{n}\left[\left(1+\frac{\delta}{\lambda_{i}}\right) I+\frac{c_{2}}{\lambda_{i}} \frac{\mathrm{d}}{\mathrm{d} u}\right] V_{2}(u ; b) \\
& =\int_{0}^{\infty} V_{2}(u+y ; b) p(y) \mathrm{d} y+\sum_{i=1}^{n} \prod_{j=i}^{n} \frac{\lambda_{j+1}+\delta}{\lambda_{j}}\left(c_{2}-c_{1}\right)
\end{aligned}
$$

Now suppose $0 \leq u \leq b$. Similar arguments as above shows that we have

$$
\begin{aligned}
& V_{1, j}(u ; b) \\
& \begin{aligned}
=e^{-\delta \mathrm{d} t} & {\left[P\left(\xi_{j+1}>\mathrm{d} t\right) E\left[V_{1, j}\left(u-c_{1} \mathrm{~d} t ; b\right)\right]\right.} \\
& \left.+P\left(\xi_{j+1} \leq \mathrm{d} t\right) E\left[V_{1, j+1}\left(u-c_{1} \mathrm{~d} t ; b\right)\right]\right]
\end{aligned}
\end{aligned}
$$

for $j=0,1, \cdots, n-2$ and

$$
\begin{aligned}
& {\left[\left(\lambda_{n}+\delta\right) I+c_{1} \frac{\mathrm{d}}{\mathrm{d} u}\right] V_{1, n-1}(u ; b)} \\
& =\lambda_{n} \int_{0}^{b-u} V_{1}(u+y ; b) p(y) \mathrm{d} y+\lambda_{n} \int_{b-u}^{\infty} V_{2}(u+y ; b) p(y) \mathrm{d} y
\end{aligned}
$$

for $j=n-1$ respectively. Substituting (8) into (9), we have (3).

Remark 2.2 Consider a compound Poisson dual model, i.e. the $W_{i}^{\prime}$ has an exponential distribution with parameter $\lambda$. When $0<u<b$, we have

$$
\begin{aligned}
& (\lambda+\delta) V_{1}(u ; b)+c_{1} \frac{\mathrm{d} V_{1}(u ; b)}{\mathrm{d} u} \\
& =\lambda \int_{0}^{b-u} V_{1}(u+y ; b) p(y) \mathrm{d} y+\lambda \int_{b-u}^{\infty} V_{2}(u+y ; b) p(y) \mathrm{d} y .
\end{aligned}
$$


When $u>b$, we have

$$
\begin{aligned}
& (\lambda+\delta) V_{2}(u ; b)+c_{2} \frac{\mathrm{d} V_{2}(u ; b)}{\mathrm{d} u} \\
& =\lambda \int_{0}^{\infty} V_{2}(u+y ; b) p(y) \mathrm{d} y+c_{2}-c_{1}
\end{aligned}
$$

Thus Theorem 2.1 generalized results obtained in A. C. Y. Ng [4].

Corollary 2.3 When $W_{i}^{\prime} s$ have generalized Erlang (2) distributions, we have

$$
\begin{aligned}
& {\left[\left(1+\frac{\delta}{\lambda_{2}}\right) I+\frac{c_{1}}{\lambda_{2}} \frac{\mathrm{d}}{\mathrm{d} u}\right]\left[\left(1+\frac{\delta}{\lambda_{1}}\right) I+\frac{c_{1}}{\lambda_{1}} \frac{\mathrm{d}}{\mathrm{d} u}\right] V_{1}(u ; b)} \\
& =\int_{0}^{b-u} V_{1}(u+y ; b) p(y) \mathrm{d} y+\int_{b-u}^{\infty} V_{2}(u+y ; b) p(y) \mathrm{d} y
\end{aligned}
$$

for $0<u<b$ and

$$
\begin{aligned}
& {\left[\left(1+\frac{\delta}{\lambda_{2}}\right) I+\frac{c_{2}}{\lambda_{2}} \frac{\mathrm{d}}{\mathrm{d} u}\right]\left[\left(1+\frac{\delta}{\lambda_{1}}\right) I+\frac{c_{2}}{\lambda_{1}} \frac{\mathrm{d}}{\mathrm{d} u}\right] V_{2}(u ; b)} \\
& =\int_{0}^{\infty} V_{2}(u+y ; b) p(y) \mathrm{d} y+\frac{1}{\lambda_{2}}\left(c_{2}-c_{1}\right) \\
& \quad+\left(1+\frac{\delta}{\lambda_{2}}\right) \frac{1}{\lambda_{1}}\left(c_{2}-c_{1}\right)
\end{aligned}
$$

for $u>b$ with the boundary conditions:

$$
\begin{gathered}
V_{1}(0 ; b)=0 \\
V_{1}(b-0 ; b)=V_{2}(b+0 ; b) \\
\left.c_{2} \frac{\mathrm{d} V_{2}(u ; b)}{\mathrm{d} u}\right|_{u=b+0}-\left.c_{1} \frac{\mathrm{d} V_{1}(u ; b)}{\mathrm{d} u}\right|_{u=b-0}=c_{2}-c_{1} \\
{\left.\left[\left(1+\frac{\delta}{\lambda_{2}}\right) I+\frac{c_{1}}{\lambda_{2}} \frac{\mathrm{d}}{\mathrm{d} u}\right]\left[\left(1+\frac{\delta}{\lambda_{1}}\right) I+\frac{c_{1}}{\lambda_{1}} \frac{\mathrm{d}}{\mathrm{d} u}\right] V_{1}(u ; b)\right|_{u=b-0}} \\
=\left[\left(1+\frac{\delta}{\lambda_{2}}\right) I+\frac{c_{2}}{\lambda_{2}} \frac{\mathrm{d}}{\mathrm{d} u}\right] \\
\times\left.\left[\left(1+\frac{\delta}{\lambda_{1}}\right) I+\frac{c_{2}}{\lambda_{1}} \frac{\mathrm{d}}{\mathrm{d} u}\right] V_{2}(u ; b)\right|_{u=b-0} \\
-\frac{1}{\lambda_{2}}\left(c_{2}-c_{1}\right)-\left(1+\frac{\delta}{\lambda_{2}}\right) \frac{1}{\lambda_{1}}\left(c_{2}-c_{1}\right) .
\end{gathered}
$$

Proof. Since ruin is immediate when $u=0$, we have (12) and (13) by the continuity condition, According to L. J. Sun [9] and Y. H. Dong et al. [10], we have $V_{21}(u ; b)=V_{11}(u ; b)$. This together with (6) and (8) yields (14). Similarly we can get (15) from (3) and (4). $\square$

Example 2.4 (Expectation of Discounted Dividends when Profits Follow an Exponential Distribution) Let profits follow an exponential distribution with $p(y)$ $=\beta e^{-\beta y}$ for $y \geq 0$. Putting the distribution function into (11) for $u>b$, we have

$$
\begin{aligned}
& {\left[\left(1+\frac{\delta}{\lambda_{2}}\right) I+\frac{c_{2}}{\lambda_{2}} \frac{\mathrm{d}}{\mathrm{d} u}\right]\left[\left(1+\frac{\delta}{\lambda_{1}}\right) I+\frac{c_{2}}{\lambda_{1}} \frac{\mathrm{d}}{\mathrm{d} u}\right] V_{2}(u ; b)} \\
& =\int_{0}^{\infty} V_{2}(u+y ; b) \beta e^{-\beta y} \mathrm{~d} y+\frac{1}{\lambda_{2}}\left(c_{2}-c_{1}\right) \\
& +\left(1+\frac{\delta}{\lambda_{2}}\right) \frac{1}{\lambda_{1}}\left(c_{2}-c_{1}\right)
\end{aligned}
$$

Applying the operator $\left(\frac{\mathrm{d}}{\mathrm{d} u}-\beta I\right)$ to both sides, we get

$$
\begin{aligned}
& \left(\frac{\mathrm{d}}{\mathrm{d} u}-\beta I\right)\left[c_{2} \frac{\mathrm{d}}{\mathrm{d} u}+(\lambda+\delta) I\right]^{2} V_{2}(u ; b) \\
& =-\lambda^{2} \beta V_{2}(u ; b)
\end{aligned}
$$

It follows that we have

$$
B_{1} \frac{\mathrm{d}^{3} V_{2}}{\mathrm{~d} u^{3}}+B_{2} \frac{\mathrm{d}^{2} V_{2}}{\mathrm{~d} u^{2}}+B_{3} \frac{\mathrm{d} V_{2}}{\mathrm{~d} u}-B_{4} V_{2}+B_{5}=0
$$

where

$$
\begin{aligned}
& B_{1}=\frac{c_{2}^{2}}{\lambda_{1} \lambda_{2}}, \\
& B_{2}=\frac{c_{2}}{\lambda_{1}}\left(1+\frac{\delta}{\lambda_{2}}\right)+\frac{c_{2}}{\lambda_{2}}\left(1+\frac{\delta}{\lambda_{1}}\right)-\beta \frac{c_{2}^{2}}{\lambda_{1} \lambda_{2}}, \\
& B_{3}=\left(1+\frac{\delta}{\lambda_{1}}\right)\left(1+\frac{\delta}{\lambda_{2}}\right)-\frac{c_{2} \beta}{\lambda_{2}}\left(1+\frac{\delta}{\lambda_{1}}\right)-\frac{c_{2} \beta}{\lambda_{1}}\left(1+\frac{\delta}{\lambda_{2}}\right), \\
& B_{4}=\beta\left(1+\frac{\delta}{\lambda_{1}}\right)\left(1+\frac{\delta}{\lambda_{2}}\right)-\beta, \\
& B_{5}=\beta\left(\frac{1}{\lambda_{2}}+\frac{1}{\lambda_{1}}+\frac{\delta}{\lambda_{1} \lambda_{2}}\right)\left(c_{2}-c_{1}\right) .
\end{aligned}
$$

The third-order linear differential equation above has a particular solution $\frac{c_{2}-c_{1}}{\delta}$. Since the characteristic equation of the differential equation

$$
B_{1} r^{3}+B_{2} r^{2}+B_{3} r-B_{4}=0
$$

has two negative roots $r_{1}$ and $r_{2}$ and a positive root $r_{3}$, we have

$$
V_{2}(u ; b)=D_{1} e^{r_{1} u}+D_{2} e^{r_{2} u}+D_{3} e^{r_{3} u}+\frac{c_{2}-c_{1}}{\delta}
$$

where $D_{1}, D_{2}$ and $D_{3}$ are constants. Similar to Andrew C.Y. Ng [7], we have $D_{1}<0, D_{2}<0$ and $D_{3}=0$. Hence we have

$$
V_{2}(u ; b)=D_{1} e^{r_{1} u}+D_{2} e^{r_{2} u}+\frac{c_{2}-c_{1}}{\delta}
$$


We put the distribution function of $p(y)=\beta e^{-\beta y}$ into (10). Then, for $b \geq u \geq 0$, we have

$$
\begin{aligned}
& {\left[\left(1+\frac{\delta}{\lambda_{2}}\right) I+\frac{c_{1}}{\lambda_{2}} \frac{\mathrm{d}}{\mathrm{d} u}\right]\left[\left(1+\frac{\delta}{\lambda_{1}}\right) I+\frac{c_{1}}{\lambda_{1}} \frac{\mathrm{d}}{\mathrm{d} u}\right] V_{1}(u ; b)} \\
& =\int_{0}^{b-u} V_{1}(u+y ; b) \beta e^{-\beta y} \mathrm{~d} y \\
& \quad+\int_{b-u}^{\infty} V_{2}(u+y ; b) \beta e^{-\beta y} \mathrm{~d} y .
\end{aligned}
$$

Applying the operator $\left(\frac{\mathrm{d}}{\mathrm{d} u}-\beta I\right)$ to both sides, we get

$$
B_{1}^{\prime} \frac{\mathrm{d}^{3} V_{1}}{\mathrm{~d} u^{3}}+B_{2}^{\prime} \frac{\mathrm{d}^{2} V_{1}}{\mathrm{~d} u^{2}}+B_{3}^{\prime} \frac{\mathrm{d} V_{1}}{\mathrm{~d} u}-B_{4}^{\prime} V_{1}=0
$$

where

$$
\begin{aligned}
& B_{1}^{\prime}=\frac{c_{1}^{2}}{\lambda_{1} \lambda_{2}}, \\
& B_{2}^{\prime}=\frac{c_{1}}{\lambda_{1}}\left(1+\frac{\delta}{\lambda_{2}}\right)+\frac{c_{1}}{\lambda_{2}}\left(1+\frac{\delta}{\lambda_{1}}\right)-\beta \frac{c_{1}^{2}}{\lambda_{1} \lambda_{2}}, \\
& B_{3}^{\prime}=\left(1+\frac{\delta}{\lambda_{1}}\right)\left(1+\frac{\delta}{\lambda_{2}}\right)-\frac{c_{1} \beta}{\lambda_{2}}\left(1+\frac{\delta}{\lambda_{1}}\right)-\frac{c_{1} \beta}{\lambda_{1}}\left(1+\frac{\delta}{\lambda_{2}}\right), \\
& B_{4}^{\prime}=\beta\left(1+\frac{\delta}{\lambda_{1}}\right)\left(1+\frac{\delta}{\lambda_{2}}\right)-\beta .
\end{aligned}
$$

Hence we have

$$
V_{1}(u ; b)=E_{1} e^{s_{1} u}+E_{2} e^{s_{2} u}+E_{3} e^{s_{3} u}
$$

where $E_{1}, E_{2}$ and $E_{3}$ are constants, $s_{1}, s_{2}$ and $s_{3}$ $\left(s_{1}<s_{2}<0<s_{3}<\beta\right)$ are the solutions of the characteristic equation

$$
B_{1}^{\prime} s^{3}+B_{2}^{\prime} s^{2}+B_{3}^{\prime} s-B_{4}^{\prime}=0
$$

Since $V(0, b)=0$, we get

$$
E_{1}+E_{2}+E_{3}=0 .
$$

Substituting back the solution for $V_{1}(u ; b)$ and $V_{2}(u ; b)$ into (16), we have

$$
\begin{aligned}
& {\left[\left(1+\frac{\delta}{\lambda_{2}}\right) I+\frac{c_{1}}{\lambda_{2}} \frac{\mathrm{d}}{\mathrm{d} u}\right]\left[\left(1+\frac{\delta}{\lambda_{1}}\right) I+\frac{c_{1}}{\lambda_{1}} \frac{\mathrm{d}}{\mathrm{d} u}\right]} \\
& {\left[E_{1} e^{s_{1} u}+E_{2} e^{s_{2} u}+E_{3} e^{s_{3} u}\right]} \\
& =\beta e^{\beta u} \int_{u}^{b} V_{1}(y ; b) \beta e^{-\beta y} \mathrm{~d} y+\beta \frac{\beta D_{1}}{\beta-r_{1}} e^{\beta u-\left(\beta-r_{1}\right) b} \\
& +\beta \frac{\beta D_{2}}{\beta-r_{2}} e^{\beta u-\left(\beta-r_{2}\right) b}+\beta \frac{C_{2}-c_{1}}{\delta} e^{-\beta(b-u)} .
\end{aligned}
$$

Since the expression above must be satisfied for all $0 \leq u \leq b$, the sum of the coefficients of $e^{\beta u}$ must be zero. Thus we have

$$
\begin{aligned}
& -\frac{E_{1} e^{s_{1} b}}{\beta-s_{1}}-\frac{E_{2} e^{s_{2} b}}{\beta-s_{2}}-\frac{E_{3} e^{s_{3} b}}{\beta-s_{3}} \\
& +\frac{D_{1} e^{r_{1} b}}{\beta-r_{1}}+\frac{D_{2} e^{r_{2} b}}{\beta-r_{2}}+\frac{c_{2}-c_{1}}{\delta}=0
\end{aligned}
$$

On the other hand, since $V_{1}(b-0 ; b)=V_{2}(b+0 ; b)$, we have

$$
\begin{aligned}
& E_{1} e^{s_{1} u}+E_{2} e^{s_{2} u}+E_{3} e^{s_{3} u} \\
& =D_{1} e^{r_{1} b}+D_{2} e^{r_{2} b}+\frac{c_{2}-c_{1}}{\delta}
\end{aligned}
$$

It follows from (10) and (11) that we have

$$
\begin{aligned}
& {\left[\left(1+\frac{\delta}{\lambda_{2}}\right) I+\frac{c_{1}}{\lambda_{2}} \frac{\mathrm{d}}{\mathrm{d} u}\right]\left[\left(1+\frac{\delta}{\lambda_{1}}\right) I+\frac{c_{1}}{\lambda_{1}} \frac{\mathrm{d}}{\mathrm{d} u}\right]} \\
& {\left[E_{1} e^{s_{1} u}+E_{2} e^{s_{2} u}+E_{3} e^{s_{3} u}\right]} \\
& =\left[\left(1+\frac{\delta}{\lambda_{2}}\right) I+\frac{c_{2}}{\lambda_{2}} \frac{\mathrm{d}}{\mathrm{d} u}\right]\left[\left(1+\frac{\delta}{\lambda_{1}}\right) I+\frac{c_{2}}{\lambda_{1}} \frac{\mathrm{d}}{\mathrm{d} u}\right] \\
& {\left[D_{1} e^{r_{1} u}+D_{2} e^{r_{2} u}+\frac{c_{2}-c_{1}}{\delta}\right]} \\
& +\frac{1}{\lambda_{2}}\left(c_{2}-c_{1}\right)+\left(1+\frac{\delta}{\lambda_{2}}\right) \frac{1}{\lambda_{1}}\left(c_{2}-c_{1}\right) .
\end{aligned}
$$

Since

$$
c_{2} V_{2}^{\prime}(b-0 ; b)-c_{1} V_{1}^{\prime}(b+0 ; b)=c_{2}-c_{1},
$$

we have

$$
\begin{aligned}
& c_{2}\left[r_{1} D_{1} e^{r_{1} b}+r_{2} D_{2} e^{r_{2} b}\right] \\
& -c_{1}\left[s_{1} E_{1} e^{s_{1} b}+s_{2} E_{2} e^{s_{2} b}+s_{3} E_{3} e^{s_{3} b}\right] \\
& =c_{2}-c_{1} .
\end{aligned}
$$

From Equations of (17), (18), (19), (20) and (21), we can get the solution of $V_{1}(u ; b)$ and $V_{2}(u ; b)$.

\section{Acknowledgements}

This work was supported by National Natural Science Foundation of China (No. 10771119). The author would like to thank Professor Chuancun Yin for his support and useful discussions.

\section{References}

[1] B. Avanzi, H. U. Gerber and E. S. W. Shiu, "Optimal Dividends in the Dual Model," Insurance: Mathematics and Economics, Vol. 41, No. 1, 2007, pp. 111-123. doi:10.1016/i.insmatheco.2006.10.002

[2] B. Avanzi and H. U. Gerber, "Optimal Dividends in the 
Dual Model with Diffusion," ASTIN Bulletin, Vol. 38, No. 2, 2008, pp. 653-667. doi:10.2143/AST.38.2.2033357

[3] H. Albrecher, A. L. Badescu and D. Landriault, "On the Dual Risk Model with Tax Payments," Insurance: Mathematics and Economics, Vol. 42, No. 3, 2008, pp. 1086-1094. doi:10.1016/j.insmatheco.2008.02.001

[4] A. C. Y. Ng, "On a Dual Model with a Dividend Threshold," Insurance: Mathematics and Economics, Vol. 44, No. 2, 2009, pp. 315-324. doi:10.1016/j.insmatheco.2008.11.011

[5] H. Cramer, "Collective Risk Theory: A Survey of the Theory from the Point of View of the Theory of Stochastic Process," Ab Nordiska Bokhandeln, Stockholm, 1955.

[6] H. L. Seal, "Stochastic Theory of a Risk Business," Wiley, New York, 1969.
[7] L. Takacs, "Combinatorial Methods in the Theory of Stochastic Processes," Wiley, New York, 1967.

[8] S. M. Li and J. Garrido, "The Gerber-Shiu Function in a Sparre Andersen Risk Process Perturbed by Diffusion," Scandinavian Actuarial Journal, Vol. 2005, No. 3, 2005, pp. 161-186. doi:10.1080/03461230510006955

[9] L. J. Sun, "The Expected Discounted Penalty at Ruin in the Erlang (2) Risk Process," Statistics and Probability Letters, Vol. 72, No. 3, 2005, pp. 205-272. doi:10.1016/j.spl.2004.12.015

[10] Y. H. Dong, G. J. Wang and Kam C. Yuen, "On the Renewal Risk Model under a Threshold Strategy," Journal of Computational and Applied Mathematics, Vol. 230, No. 1, 2009, pp. 22-33. doi:10.1016/j.cam.2008.10.049 